\title{
Quantum Latin squares and unitary error bases
}

\author{
Benjamin Musto \\ Jamie Vicary \\ benjamin.musto@cs.ox.ac.uk jamie.vicary@cs.ox.ac.uk \\ Department of Computer Science, University of Oxford
}

Friday $19^{\text {th }}$ August, 2016

\begin{abstract}
In this paper we introduce quantum Latin squares, combinatorial quantum objects which generalize classical Latin squares, and investigate their applications in quantum computer science. Our main results are on applications to unitary error bases (UEBs), basic structures in quantum information which lie at the heart of procedures such as teleportation, dense coding and error correction. We present a new method for constructing a UEB from a quantum Latin square equipped with extra data. Developing construction techniques for UEBs has been a major activity in quantum computation, with three primary methods proposed: shift-andmultiply, Hadamard, and algebraic. We show that our new approach simultaneously generalizes the shift-and-multiply and Hadamard methods. Furthermore, we explicitly construct a UEB using our technique which we prove cannot be obtained from any of these existing methods.
\end{abstract}

\section{Introduction}

We begin with the definition of a quantum Latin square.

Definition 1. A quantum Latin square of order $n$ is an $n$-by-n array of elements of the Hilbert space $\mathbb{C}^{n}$, such that every row and every column is an orthonormal basis.

Example 2. Here is a quantum Latin square given in terms of the computational basis elements $\{|0\rangle,|1\rangle,|2\rangle,|3\rangle\} \subset \mathbb{C}^{4}$ :

\begin{tabular}{|c|c|c|c|}
\hline$|0\rangle$ & $|1\rangle$ & $|2\rangle$ & $|3\rangle$ \\
\hline$\frac{1}{\sqrt{2}}(|1\rangle-|2\rangle)$ & $\frac{1}{\sqrt{5}}(i|0\rangle+2|3\rangle)$ & $\frac{1}{\sqrt{5}}(2|0\rangle+i|3\rangle)$ & $\frac{1}{\sqrt{2}}(|1\rangle+|2\rangle)$ \\
\hline$\frac{1}{\sqrt{2}}(|1\rangle+|2\rangle)$ & $\frac{1}{\sqrt{5}}(2|0\rangle+i|3\rangle)$ & $\frac{1}{\sqrt{5}}(i|0\rangle+2|3\rangle)$ & $\frac{1}{\sqrt{2}}(|1\rangle-|2\rangle)$ \\
\hline$|3\rangle$ & $|2\rangle$ & $|1\rangle$ & $|0\rangle$ \\
\hline
\end{tabular}

It can readily be checked that along each row, and along each column, the elements form an orthonormal basis for $\mathbb{C}^{4}$. We can compare this to the classical notion of Latin square [11]. 
Definition 3. A classical Latin square of order $n$ is an $n$-by- $n$ array of integers in the range $\{0, \ldots, n-1\}$, such that every row and column contains each number exactly once.

By interpreting a number $k \in\{0, \ldots, n-1\}$ as a computational basis element $|k\rangle \in \mathbb{C}^{n}$, we can turn an array of numbers into an array of Hilbert space elements:

\begin{tabular}{|l|l|l|l|}
\hline 3 & 1 & 0 & 2 \\
\hline 1 & 0 & 2 & 3 \\
\hline 2 & 3 & 1 & 0 \\
\hline 0 & 2 & 3 & 1 \\
\hline
\end{tabular}

\begin{tabular}{|l|l|l|l|}
\hline$|3\rangle$ & $|1\rangle$ & $|0\rangle$ & $|2\rangle$ \\
\hline$|1\rangle$ & $|0\rangle$ & $|2\rangle$ & $|3\rangle$ \\
\hline$|2\rangle$ & $|3\rangle$ & $|1\rangle$ & $|0\rangle$ \\
\hline$|0\rangle$ & $|2\rangle$ & $|3\rangle$ & $|1\rangle$ \\
\hline
\end{tabular}

It is easy to see that the original array of numbers is a classical Latin square if and only if the corresponding grid of Hilbert space elements is a quantum Latin square. However, as Example 2 makes clear, not every quantum Latin square is of this form.

Our main results are on the construction of unitary error bases (UEBs) [14], also known as unitary operator bases. These are basic structures in quantum information which play a central role in quantum teleportation [6], dense coding [13] and error correction [18]. Since UEBs are hard to find, and given their wide applicability, construction techniques for UEBs have been widely studied [12, 14, 15, 21]. In this paper, we propose a new method for construction of UEBs:

- Quantum shift-and-multiply method (QSM). Requires a quantum Latin square and a family of Hadamard matrices. (See Definition 18.)

We compare this to the other methods that have been proposed in the literature:

- Shift-and-multiply method (SM). Requires a classical Latin square and a family of Hadamard matrices. (See Definition 21,)

- Hadamard method (HAD). Requires a pair of mutually-unbiased bases. (See Definition 33.)

- Algebraic method (ALG). Requires a finite group equipped with a projective representation, satisfying certain properties. (See Definition 41.)

Our theorems concern the relationships between these constructions. In Theorems 22 and 34, we prove that QSM contains SM and HAD as special cases. We also use QSM to construct a concrete unitary error basis $\mathcal{M}$ (Example 19), and prove that it is not equivalent to one arising from SM, HAD or ALG (Corollaries 32, 39] and 44] respectively.)

The relationships between these constructions, up to a standard notion of equivalence of UEBs (see Definition 16), are indicated by the following Venn diagram:

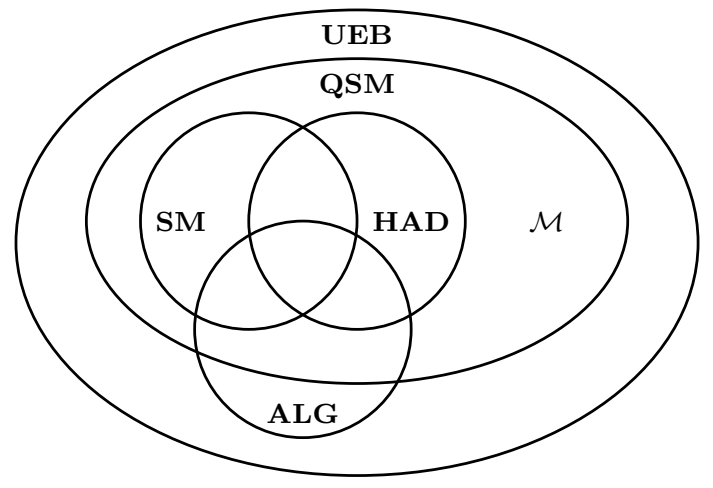


Our work strongly extends previous results, in an area that has not seen progress since 2003. But there is much still to be settled: in particular, we do not know whether ALG is a subset of QSM, or whether QSM equals UEB.

Categorical quantum mechanics is a research programme in which powerful techniques of monoidal category theory are used to understand quantum computational phenomena [1, 2, 8], using a graphical notation which can make the high-level structure of computations easier to understand. The main results of this paper were originally developed using this approach (see also [17]), although we have chosen to present them here in a conventional way. We feel this is a good advert for the power of categorical quantum mechanics; certainly, we could not have developed our results without using these techniques.

There are interesting connections between Hadamard matrices, unitary error bases and quantum Latin squares. In Section 2 we show that a quantum Latin square can be constructed from any Hadamard matrix. Hadamard matrices are mathematically equivalent to the data for a pair of mutually unbiased bases [5], the study and classification of which is a major activity in quantum computer science [4, 10, 16, 19]. It has also been shown that in some cases a family of mutually unbiased bases can be extracted from a UEB [3]. So quantum Latin squares can be built from Hadamards, which can be built from UEBs, which can be built from quantum Latin squares; an interesting tapestry of results for which we currently lack a good intuition.

Acknowledgements. The authors are grateful to Dominic Verdon for useful discussions, and to EPSRC for financial support.

\section{Quantum Latin squares from Hadamard matrices}

In this section we introduce some basic properties of quantum Latin squares, show how to construct a quantum Latin square from a Hadamard matrix, and prove that our quantum Latin square of Example 2 is not equivalent to one arising in this way.

We begin by developing a precise notation for working with quantum Latin squares. Throughout, we assume we are working with a quantum Latin square of order $n$, and that indices $i, j, k, p, q$ range from 0 to $n-1$.

Definition 4. For a quantum Latin square $Q$, we define the following:

- $Q_{i}$ is the matrix whose columns are the entries of the $i$ th row of $Q$;

- $Q_{i j} \in \mathbb{C}^{n}$ is the Hilbert space element at the $i$ th row and $j$ th column of $Q$;

- $Q_{i j k}:=\left(Q_{i j}\right)_{k}=\left\langle k \mid Q_{i j}\right\rangle \in \mathbb{C}$ is the coefficient of the basis vector $|k\rangle$.

For a matrix $M$, it is a standard notation to write $M_{i j}$ for the element at the $i$ th row and $j$ th column. Combining this with Definition 4 , we have the following:

$$
\left(Q_{i}\right)_{j k}=Q_{i k j}
$$

Note that the order of the final two indices changes.

Given a collection of numbers $Q_{i j k} \in \mathbb{C}$, we can easily identify when they arise from a quantum Latin square. For a matrix $M$, we write $M^{*}$ for the conjugate matrix, $M^{\mathrm{T}}$ for the transpose matrix, and $M^{\dagger}=\left(M^{*}\right)^{\dagger}=\left(M^{\dagger}\right)^{*}$ for the conjugate transpose matrix. 
Lemma 5. A family of numbers $Q_{i j k} \in \mathbb{C}$ arise from a quantum Latin square if and only if they satisfy the following properties for all $i, p, q$ :

$$
\begin{aligned}
& \sum_{j} Q_{i p j}^{*} Q_{i q j}=\delta_{p q}, \text { or equivalently the matrices } Q_{i} \text { are unitary } \\
& \sum_{j} Q_{p i j}^{*} Q_{q i j}=\delta_{p q}
\end{aligned}
$$

Proof. Equations (44) and (5) are exactly the condition that the rows and columns, respectively, of the quantum Latin square form orthonormal bases. Unitarity of $Q_{i}$ means precisely $\left(Q_{i}^{\dagger} \circ Q_{i}\right)_{p q}=\delta_{p q}$, which expands to $\sum_{j}\left(Q_{i}^{\dagger}\right)_{p j}\left(Q_{i}\right)_{j q}=\sum_{j} Q_{i p j}^{*} Q_{i q j}=\delta_{p q}$. (Recall that for an operator $Q$ on a finite-dimensional Hilbert space, $Q \circ Q^{\dagger}=\mathbb{I}_{n}$ if and only if $Q^{\dagger} \circ Q=\mathbb{I}_{n}$.)

The condition (5) equivalently says that the matrices formed by the columns of the Latin square are unitary, but this is not a fact that we will need directly.

There are certain trivial ways to transform a quantum Latin square into a different quantum Latin square, which we use to define a notion of equivalence.

Definition 6. Two quantum Latin squares are equivalent when one can be obtained from the other by permuting rows and columns, multiplying rows and columns by unit complex numbers, and applying a fixed unitary to every element. Algebraically, quantum Latin squares $Q$ and $Q^{\prime}$ are equivalent when there exists some unitary $U$, diagonal unitary $D$, permutation matrix $P$, permutation $\phi$, and a family of unit complex numbers $c_{j}$, such that the following holds:

$$
Q_{j}^{\prime}=c_{j} U \circ Q_{\phi(j)} \circ P \circ D
$$

We now give the standard definition of a Hadamard matrix, as a square matrix with entries of absolute value 1 which is proportional to a unitary matrix.

Definition 7 (See [20], Definition 2.1). A Hadamard matrix of order $n$ is an $n$-by-n matrix $H$ with the following properties for all $i, j$, which we write in both matrix and index form:

$$
\begin{aligned}
\left|H_{i j}\right| & =1 & H_{i j} H_{i j}^{*} & =1 \\
H \circ H^{\dagger} & =n \mathbb{I}_{n} & \sum_{p} H_{i p} H_{j p}^{*} & =n \delta_{i j} \\
H^{\dagger} \circ H & =n \mathbb{I}_{n} & \sum_{p} H_{p i}^{*} H_{p j} & =n \delta_{i j}
\end{aligned}
$$

Definition 8 (See [21], Section 4). Two Hadamard matrices are equivalent when one can be obtained from the other by permuting rows and columns, and multiplying rows and columns by unit complex numbers. Algebraically, $H, H^{\prime}$ are equivalent if there exist $P_{1}, P_{2}$ permutation matrices and $D_{1}, D_{2}$ unitary diagonal matrices such that:

$$
H^{\prime}=D_{1} \circ P_{1} \circ H \circ P_{2} \circ D_{2}
$$

We now give the construction of a quantum Latin square from a Hadamard matrix.

Definition 9. For a square matrix $M$, let $\operatorname{diag}(M, i)$ be the diagonal matrix whose diagonal entries are given by the $i$ th row of $M$ :

$$
\operatorname{diag}(M, i)_{j k}:=\delta_{j k} M_{i j}
$$


Definition 10. For a Hadamard matrix $H$ of order $n$, its associated quantum Latin square $Q_{H}$ of order $n$ is defined as follows:

$$
\left(Q_{H}\right)_{j}:=\frac{1}{n} H \circ \operatorname{diag}(H, j)^{\dagger} \circ H^{\dagger}
$$

We will refer to a quantum Latin square constructed in this way as a Hadamard quantum Latin square.

Theorem 11. The associated quantum Latin square construction is correct.

Proof. To establish property (4), we note that $\left(Q_{H}\right)_{j}$ is the composite of three unitary matrices, and is therefore unitary. To verify (5), we write expression (12) in index form:

$$
\begin{aligned}
& \left(Q_{H}\right)_{q i j} \stackrel{\text { 哥 }}{(}\left(\left(Q_{H}\right)_{q}\right)_{j i} \stackrel{\underline{\underline{112}}}{=} \frac{1}{n} \sum_{r s} H_{j r} \operatorname{diag}(H, q)_{r s}^{\dagger} H_{s i}^{\dagger} \\
& \stackrel{111}{=} \frac{1}{n} \sum_{r s} H_{j r} H_{q r}^{*} \delta_{r s} H_{i s}^{*}=\frac{1}{n} \sum_{r} H_{j r} H_{q r}^{*} H_{i r}^{*}
\end{aligned}
$$

We then perform the following calculation:

$$
\begin{aligned}
& \sum_{j}\left(Q_{H}\right)_{p i j}^{*}\left(Q_{H}\right)_{q i j} \stackrel{\sqrt[13]{=}}{=} \frac{1}{n^{2}} \sum_{j}\left(\sum_{r} H_{j r}^{*} H_{p r} H_{i r}\right)\left(\sum_{s} H_{j s} H_{q s}^{*} H_{i s}^{*}\right) \\
& =\frac{1}{n^{2}} \sum_{r s}\left(\sum_{j} H_{j r}^{*} H_{j s}\right) H_{p r} H_{i r} H_{q s}^{*} H_{i s}^{*} \stackrel{\underline{\underline{G}}}{=} \frac{1}{n} \sum_{r s} \delta_{r s} H_{p r} H_{i r} H_{q s}^{*} H_{i s}^{*} \\
& =\frac{1}{n} \sum_{r} H_{p r} H_{q r}^{*} H_{i r} H_{i r}^{*} \stackrel{\underline{\underline{\theta}}}{=} \frac{1}{n} \sum_{r} H_{p r} H_{q r}^{*} \stackrel{\underline{\underline{\theta}}}{=} \delta_{p q}
\end{aligned}
$$

In the second equality here, the sum is being reorganized.

We now establish a lemma which we will use to prove Lemma 13 and later Proposition 36 .

Lemma 12. Let $p$ be the permutation associated with the permutation matrix $P$ such that $P=\sum_{k}|p(k)\rangle\langle k|$ and $D$ be a diagonal unitary. Then the following equations hold:

$$
\begin{aligned}
\operatorname{diag}(P \circ H, i) & =\operatorname{diag}(H, p(i))=\operatorname{diag}\left(H_{p(i), 0}, \ldots, H_{p(i), n-1}\right) \\
\operatorname{diag}(H \circ P, i) & =\operatorname{diag}\left(H_{i, p(0)}, \ldots, H_{i, p(n-1)}\right) \\
\operatorname{diag}(D \circ H, i) & =D_{i i} \operatorname{diag}(H, i) \\
\operatorname{diag}(H \circ D, i) & =D \circ \operatorname{diag}(H, i)=\operatorname{diag}(H, i) \circ D
\end{aligned}
$$

Proof. Straightforward calculation.

Lemma 13. Equivalent Hadamards give rise to equivalent quantum Latin squares.

Proof. We will prove equivalence on a case-by-case basis. Suppose $H^{\prime}=P \circ H$. Then we have the following, where we use the fact that $P^{-1}=P^{\dagger}=P^{T}$ :

$$
\begin{aligned}
& \left(Q_{H^{\prime}}\right)_{j} \stackrel{(12)}{=} \frac{1}{n} P \circ H \circ \operatorname{diag}(P \circ H, j)^{\dagger} \circ H^{\dagger} \circ P^{-1} \\
& \text { (15) } \frac{1}{n} P \circ H \circ \operatorname{diag}(H, p(j))^{\dagger} \circ H^{\dagger} \circ P^{-1} \\
& \stackrel{\text { 自 }}{\sim} \frac{1}{n} H \circ \operatorname{diag}(H, j)^{\dagger} \circ H^{\dagger} \stackrel{\sqrt{\underline{12}}}{=}\left(Q_{H}\right)_{j}
\end{aligned}
$$

We now consider $H^{\prime}=H \circ P$ :

$$
\begin{aligned}
\left(Q_{H^{\prime}}\right)_{j} & \stackrel{\sqrt{12}}{=} \frac{1}{n} H \circ P \circ \operatorname{diag}(H \circ P, j)^{\dagger} \circ P^{-1} \circ H^{\dagger} \\
& \stackrel{\underline{\underline{\underline{16}}}}{\frac{1}{n}} H \circ P \circ \operatorname{diag}\left(H_{j, p(0)}, \ldots, H_{j, p(n-1)}\right)^{\dagger} \circ P^{-1} \circ H^{\dagger} \\
& \stackrel{(27)}{=} \frac{1}{n} H \circ P \circ P^{-1} \circ \operatorname{diag}(H, j)^{\dagger} \circ H^{\dagger} \\
& =\frac{1}{n} H \circ \operatorname{diag}(H, j)^{\dagger} \circ H^{\dagger} \stackrel{\underline{\underline{122}}}{=}\left(Q_{H}\right)_{j}
\end{aligned}
$$


Finally, suppose $H^{\prime}=D_{1} \circ H \circ D_{2}$, with $D_{1}=\operatorname{diag}\left(c_{1}, \ldots, c_{n}\right)$, where $\left|c_{i}\right|=1$. Then we calculate as follows:

$$
\begin{aligned}
& \left(Q_{H^{\prime}}\right)_{j} \stackrel{(12)}{=} \frac{1}{n} D_{1} \circ H \circ D_{2} \circ \operatorname{diag}\left(D_{1} \circ H \circ D_{2}, j\right)^{\dagger} D_{2}^{\dagger} \circ H^{\dagger} \circ D_{1}^{\dagger} \\
& \stackrel{\text { I17] }}{=} \frac{1}{n} D_{1} \circ H \circ D_{2} \circ c_{j} \operatorname{diag}\left(H \circ D_{2}, j\right)^{\dagger} \circ D_{2}^{\dagger} \circ H^{\dagger} \circ D_{1} \\
& \stackrel{[18]}{=} \frac{1}{n} D_{1} \circ H \circ D_{2} \circ c_{j} \operatorname{diag}(H, j)^{\dagger} D_{2} \circ D_{2}^{\dagger} \circ H^{\dagger} \circ D_{1} \\
& =\frac{1}{n} D_{1} \circ H \circ D_{2} \circ c_{j} \operatorname{diag}(H, j)^{\dagger} \circ H^{\dagger} \circ D_{1} \\
& \stackrel{\text { [6] }}{\sim} \frac{1}{\sqrt{n}} \operatorname{diag}(H, j)^{\dagger} \circ H^{\dagger} \\
& \stackrel{[6]}{\sim} \frac{1}{n} H \circ \operatorname{diag}(H, j)^{\dagger} \circ H^{\dagger} \stackrel{\sqrt{12]}}{=}\left(Q_{H}\right)_{j}
\end{aligned}
$$

This completes the proof.

Finally, we prove that our example quantum Latin square does not arise in this way, even up to equivalence. This makes use of some results that we prove later in the paper.

Proposition 14. The quantum Latin square given in Example 2 is not equivalent to a quantum Latin square constructed from a Hadamard.

Proof. Let $H_{\alpha}$ be the family of Hadamard matrices as defined in equation (32), let $\left(Q_{H_{\alpha}}\right)_{j}:=\frac{1}{n} H_{\alpha} \circ \operatorname{diag}\left(H_{\alpha}, j\right)^{\dagger} \circ H_{\alpha}^{\dagger}$ be the associated quantum Latin squares, and let $Q$ be the quantum Latin square of Example 2, By Lemma 13 and Proposition 37, any quantum Latin square arising from a Hadamard matrix in the manner of Definition 10 is equivalent to $Q_{H_{\alpha}}$ for some value of $\alpha$.

For a contradiction, suppose that $Q$ and $Q_{H_{\alpha}}$ are equivalent in the manner of Definition 6, for some fixed value of $\alpha$. So there exists some unitary matrix $U$, diagonal unitary matrix $D$, permutation matrix $P$, permutation $\phi$, and a family of unit complex numbers $c_{j}$, such that the following holds:

$$
\left(Q_{H_{\alpha}}\right)_{j}=c_{j} U \circ Q_{\phi(j)} \circ P \circ D
$$

Note that the composite $P \circ D$ is unitary; so the families of matrices $\left(Q_{H_{\alpha}}\right)_{j}$ and $Q_{j}$, which are unitary by Lemma 5, are equivalent families in the sense of Definition 16.

The family $\left(Q_{H_{\alpha}}\right)_{j}$ are simultaneously monomializable, by the matrix $Y$ defined in equation (33). (This follows from Theorem 38, in which we show that the members of $\mathcal{F}_{\alpha}$, which include the $\left(Q_{H_{\alpha}}\right)_{j}$ as a subset, are simultaneously monomializable.) So all together, the family of matrices $Q_{j}$ contains the identity, and is equivalent in the sense of Definition 16 to a monomial family. So by Proposition 28, the family $Q_{j}$ is simultaneously monomializable, and thus by Proposition 29, their 12th powers must all commute. But as established in the proof of Theorem [30, the 12th powers of $Q_{1}=\mathcal{M}_{01}$ and $Q_{2}=\mathcal{M}_{02}$ do not commute. This gives us our contradiction.

\section{$3 \quad$ Unitary error bases from quantum Latin squares}

In this section we define unitary error bases, and present our new quantum shift-andmultiply construction, which produces a unitary error basis from a quantum Latin square equipped with a family of Hadamard matrices. We then introduce an example UEB $\mathcal{M}$, which will play an important role in later sections where we show that it cannot arise from the shift-and-multiply, Hadamard or algebraic methods, even up to equivalence.

We begin with the definition of unitary error basis. As remarked in the introduction, these structures play a central role in quantum computation. 
Definition 15 (See [14], Section 1). For a Hilbert space $H$ of dimension $n$, a unitary error basis (or unitary operator basis) is a family of $n^{2}$ unitary matrices $U_{i j}: H \rightarrow H$ which form an orthogonal basis:

$$
\operatorname{Tr}\left(U_{i j}^{\dagger} \circ U_{i^{\prime} j^{\prime}}\right)=\delta_{i i^{\prime}} \delta_{j j^{\prime}} n
$$

There is a standard notion of equivalence of unitary error bases, which we recall here.

Definition 16 (See [14], Section 2). Two families of unitary matrices $\mathcal{A}, \mathcal{B}$ are equivalent if there are unitary matrices $U$ and $V$, such that for any element $A \in \mathcal{A}$, there is an element $B \in \mathcal{B}$ and a unit complex number $c$ such that the following holds:

$$
B=c U \circ A \circ V
$$

The following technical lemma will be useful later.

Lemma 17. Let $D$ be a diagonal matrix, and $A$ be a square matrix which is zero along the main diagonal, such that $D$ and $A$ are composable. Then $D \circ A$ is zero along the main diagonal.

Proof. We perform the following calculation of the diagonal elements of $D \circ A$ :

$$
(D \circ A)_{i i}=\sum_{k} D_{i k} A_{k i}=\sum_{k} \delta_{i k} D_{i i} A_{k i}=D_{i i} A_{i i}=0
$$

Here we apply the definition of matrix composition, the diagonal property of $D$, the properties of the sum, and the hypothesis that $A$ is zero along the main diagonal.

We now define the main construction of focus in this paper. This construction is similar to Werner's shift-and-multiply method [21], the difference being that ours is in terms of quantum Latin squares. As usual, we take all indices in the range 0 to $n-1$.

Definition 18 (Quantum shift-and-multiply method). Let $Q$ be a quantum Latin square of order $n$, and $H_{j}$ be a family of $n$ Hadamard matrices of order $n$. Then the associated quantum shift-and-multiply basis has the following elements:

$$
S_{i j}:=Q_{j} \circ \operatorname{diag}\left(H_{j}, i\right)
$$

In words, the $(i, j)$ entry of the quantum shift-and-multiply basis is the matrix given by the $j$ th row of the quantum Latin square, composed with the diagonal matrix formed from the $i$ th row of the $j$ th Hadamard matrix.

We illustrate this with an example. This example will play a central role, as we will show in the remainder of the paper that it cannot be obtained, even up to equivalence, by any of the existing methods of unitary error basis construction.

Example 19. The quantum shift-and-multiply basis $\mathcal{M}$ is constructed from the quantum Latin square of Example 2, and from the following family of Hadamard matrices:

$$
H_{0}=H_{1}=H_{2}=H_{3}=\left(\begin{array}{cccc}
1 & 1 & 1 & 1 \\
1 & i & -1 & -i \\
1 & -1 & 1 & -1 \\
1 & -i & -1 & i
\end{array}\right)
$$

The resulting family of 16 matrices is listed in Appendix A.1. 
We now show that quantum shift-and-multiply bases are unitary error bases. This has similarities with Werner's original proof [21] for standard shift-and-multiply bases (see Section 4), but our use of quantum Latin squares requires nontrivial extra ideas.

Theorem 20. Quantum shift-and-multiply bases are unitary error bases.

Proof. First, we note that the elements $S_{i j}=Q_{j} \circ \operatorname{diag}\left(H_{j}, i\right)$ are unitary, since they are composites of unitary matrices: the matrix $Q_{j}$ is the $j$ th row of a quantum Latin square, and hence unitary by Lemma 5, and $\operatorname{diag}\left(H_{j}, i\right)$ is a diagonal matrix with unit complex numbers along the diagonal, and hence unitary.

We must establish the following trace property:

$$
\operatorname{Tr}\left(S_{i j}^{\dagger} \circ S_{i^{\prime} j^{\prime}}\right)=n \delta_{i i^{\prime}} \delta_{j j^{\prime}}
$$

We first consider the case that $j=j^{\prime}$ and $i=i^{\prime}$. By unitarity of $S_{i j}$ we have $S_{i j} \circ S_{i^{\prime} j^{\prime}}^{\dagger}=\mathbb{I}_{n}$, with $\operatorname{Tr}\left(\mathbb{I}_{n}\right)=n$, and so the condition follows.

Next we consider the case that $j=j^{\prime}$ and $i \neq i^{\prime}$. We perform the following calculation:

$$
\begin{aligned}
\operatorname{Tr}\left(S_{i j}^{\dagger} \circ S_{i^{\prime} j}\right) & \stackrel{(22)}{=} \operatorname{Tr}\left(\operatorname{diag}\left(H_{j}, i\right)^{\dagger} \circ Q_{j}^{\dagger} \circ Q_{j} \circ \operatorname{diag}\left(H_{j}, i^{\prime}\right)\right) \\
& \stackrel{\underline{\underline{4}}}{=} \operatorname{Tr}\left(\operatorname{diag}\left(H_{j}, i\right)^{\dagger} \circ \operatorname{diag}\left(H_{j}, i^{\prime}\right)\right)
\end{aligned}
$$

The final expression is equal to the inner product of rows $i$ and $i^{\prime}$ of the Hadamard $H_{j}$. Since distinct rows of a Hadamard are orthogonal, the result is zero as required.

It remains to consider the case that $j \neq j^{\prime}$. We use the cyclic property of the trace to rearrange our trace expression:

$$
\begin{array}{r}
\operatorname{Tr}\left(S_{i j}^{\dagger} \circ S_{i^{\prime} j^{\prime}}\right) \stackrel{\sqrt[222]{=}}{=} \operatorname{Tr}\left(\operatorname{diag}\left(H_{j}, i\right)^{\dagger} \circ Q_{j}^{\dagger} \circ Q_{j^{\prime}} \circ \operatorname{diag}\left(H_{j^{\prime}}, i^{\prime}\right)\right) \\
=\operatorname{Tr}\left(\operatorname{diag}\left(H_{j^{\prime}}, i^{\prime}\right) \circ \operatorname{diag}\left(H_{j}, i\right)^{\dagger} \circ Q_{j}^{\dagger} \circ Q_{j^{\prime}}\right)
\end{array}
$$

Inside the trace there is the composite $\operatorname{diag}\left(H_{j^{\prime}}, i^{\prime}\right) \circ \operatorname{diag}\left(H_{j}, i\right)^{\dagger}$, which is diagonal. There is also $Q_{j}^{\dagger} \circ Q_{j^{\prime}}$, which by the following argument is zero along the diagonal:

$$
\left(Q_{j}^{\dagger} \circ Q_{j^{\prime}}\right)_{k k}=\sum_{l}\left(Q_{j}^{\dagger}\right)_{k l}\left(Q_{j^{\prime}}\right)_{l k}=\sum_{l}\left(Q_{j}^{*}\right)_{l k}\left(Q_{j^{\prime}}\right)_{l k} \stackrel{\underline{\underline{\underline{3}}}}{=} \sum_{l} Q_{j k l}^{*} Q_{j^{\prime} k l} \stackrel{\underline{\underline{\underline{5}}}}{=} \delta_{j j^{\prime}}=0
$$

Hence by Lemma 17, expression (25) is zero as required.

\section{Shift-and-multiply method}

The shift-and-multiply method of Werner [21], which was a direct inspiration for our own results, can straightforwardly be seen as a special case of our quantum shift-andmultiply method. Our focus in this section is the proof that the unitary error basis $\mathcal{M}$ of Example 19 is not equivalent to a shift-and-multiply basis, and thus that the shift-andmultiply bases are strictly contained within the quantum shift-and-multiply bases.

Definition 21. A shift-and-multiply basis is a quantum shift-and-multiply basis where the quantum Latin square is a classical Latin square.

Theorem 22. Every shift-and-multiply basis is a quantum shift-and-multiply basis.

Proof. Follows immediately from Definitions 3 and 21. 
Monomial matrices will be crucial to our proof strategy.

Definition 23. A monomial matrix is a square matrix with exactly one nonzero entry in each row and each column. Equivalently, it is any matrix $A$ which can be expressed as $A=D_{A} \circ P_{A}$, where $D_{A}$ is a diagonal matrix and $P_{A}$ is a permutation matrix.

Lemma 24. Let $p$ be a permutation, $P=\sum_{k}|p(k)\rangle\langle k|$ be the corresponding permutation matrix, and $D=\sum_{k} d_{k}|k\rangle\langle k|$ and $D^{\prime}=\sum_{k} d_{p(k)}|k\rangle\langle k|$ be diagonal matrices. Then the following holds:

$$
D \circ P=P \circ D^{\prime}
$$

Proof. We perform the following calculation:

$$
\begin{aligned}
& D \circ P=P \circ P^{\dagger} \circ D \circ P=P \circ\left(\sum_{i j k}|i\rangle\left\langle p(i)\left|d_{j}\right| j\right\rangle\langle j \mid p(k)\rangle\langle k|\right) \\
& =P \circ\left(\sum_{i k} d_{p(k)}|i\rangle\langle p(i) \mid p(k)\rangle\langle k|\right)=P \circ\left(\sum_{i} d_{p(i)}|i\rangle\langle i|\right)=P \circ D^{\prime}
\end{aligned}
$$

This completes the proof.

Lemma 25. The set of monomial matrices is closed under composition, taking inverses, taking adjoints, and multiplication by nonzero complex scalars.

Proof. Straightforward.

Definition 26. A square matrix $A$ is monomializable if there exists a unitary matrix $U$ such that $U \circ A \circ U^{\dagger}$ is monomial.

Definition 27. A family of square matrices $A_{1}, \ldots, A_{n}$ are simultaneously monomializable if they are all monomializable by the same unitary matrix $U$.

We establish the following propositions, the first of which is adapted and generalized to suit our purposes from the literature.

Proposition 28 (See [14], final part of the proof of Theorem 3). If a family $\mathcal{S}$ of unitary matrices containing the identity is equivalent (in the sense of Definition 16) to a family of monomial matrices, then the members of $\mathcal{S}$ are simultaneously monomializable.

Proof. Let $\mathcal{S}=\left\{S_{i}\right\}$ be a family of unitary matrices with $S_{0}=\mathbb{I}_{n}$. Suppose $S_{i}$ is equivalent to some monomial family $\mathcal{T}=\left\{T_{i}\right\}$ with $T_{i}=c_{i} U S_{i} V$, such that each $c_{i}$ is a complex number of norm 1 , and $U, V$ are unitary matrices. We then perform the following calculation:

$$
\frac{c_{0}}{c_{j}} T_{j} T_{0}^{\dagger}=\frac{c_{0}}{c_{j}}\left(c_{j} U S_{j} V\right)\left(c_{0} U S_{0} V\right)^{\dagger}=c_{0} c_{0}^{*} U S_{j} V V^{\dagger} \mathbb{I}_{n} U^{\dagger}=U S_{j} U^{\dagger}
$$

The left hand side is monomial by Lemma 25, and hence $U$ simultaneously monomializes $S_{i}$.

Proposition 29. Let $A, B$ be square matrices of size $n$, and let $\mu_{n}$ be the lowest common multiple of $\{1,2, \ldots, n\}$. If $A$ and $B$ are simultaneously monomializable, then $A^{\mu_{n}}$ and $B^{\mu_{n}}$ commute. 
Proof. Suppose $A, B$ are simultaneously monomializable, with $\mu_{n}$ defined as above. Then there exists a unitary matrix $U$ such that $U A U^{\dagger}=D_{A} P_{A}$ and $U B U^{\dagger}=D_{B} P_{B}$ where $D_{A}, D_{B}$ are diagonal matrices and $P_{A}, P_{B}$ are permutation matrices. Note that $A=U^{\dagger} D_{A} P_{A} U$, so we have the following:

$$
A^{\mu_{n}}=U^{\dagger}\left(D_{A} P_{A}\right)^{\mu_{n}} U=U^{\dagger} \widetilde{D}_{A} P_{A}^{\mu_{n}} U
$$

Here $\widetilde{D}_{A}$ is some diagonal matrix, and the last equality is obtained by repeated application of Lemma 24 and the fact that diagonal matrices are closed under composition. Since $P_{A}$ is a permutation matrix of dimension $n$ it has order $k$, where $k$ is the lowest common multiple of the lengths of the permutation's cycles. Each cycle has length $\in\{1,2, \ldots, n\}$. Thus $k$ divides $\mu_{n}$, and so $P_{A}^{\mu_{n}}=\mathbb{I}_{n}$. So $A^{\mu_{n}}=U^{\dagger} \widetilde{D}_{A} U$, and by the same argument, $B^{\mu_{n}}=U^{\dagger} \widetilde{D}_{B} U$ for some diagonal matrix $\widetilde{D}_{B}$. We then demonstrate that $A^{\mu_{n}}$ and $B^{\mu_{n}}$ commute:

$$
A^{\mu_{n}} B^{\mu_{n}}=U^{\dagger} \widetilde{D}_{A} U U^{\dagger} \widetilde{D}_{B} U=U^{\dagger} \widetilde{D}_{A} \widetilde{D}_{B} U=U^{\dagger} \widetilde{D}_{B} \widetilde{D}_{A} U=U^{\dagger} \widetilde{D}_{B} U U^{\dagger} \widetilde{D}_{A} U=B^{\mu_{n}} A^{\mu_{n}}
$$

The central equality here holds because diagonal matrices commute.

We are now ready to prove the necessary properties of our example basis.

Theorem 30. The basis $\mathcal{M}$ of Example 19 is not equivalent to a monomial basis.

Proof. For a contradiction, suppose that $\mathcal{M}$ is equivalent to a monomial basis. Note that $\mathcal{M}$ contains the identity matrix, so by Proposition 28 the elements of the UEB are simultaneously monomializable. The least common multiple of $\{1,2,3,4\}$ is $\mu_{4}=12$; thus by Proposition 29 the 12 th powers of the elements of $\mathcal{M}$ will commute. To exhibit the contradiction, we compute the following commutator:

$$
\left(\mathcal{M}_{01}\right)^{12}\left(\mathcal{M}_{02}\right)^{12}-\left(\mathcal{M}_{02}\right)^{12}\left(\mathcal{M}_{01}\right)^{12}=\frac{12168}{15625}\left(\begin{array}{cccc}
-i & 0 & 0 & 2 \\
0 & 0 & 0 & 0 \\
0 & 0 & 0 & 0 \\
-2 & 0 & 0 & i
\end{array}\right) \neq 0
$$

This completes the proof.

Proposition 31. Shift-and-multiply bases are monomial bases.

Proof. Recall from Definition 21 of a shift-and-multiply basis that each matrix is the product of a diagonal matrix with the permutation matrix given by a row of a classical Latin square. By definition, the result is a monomial matrix.

Corollary 32. The basis $\mathcal{M}$ of Example 19 is not equivalent to a shift-and-multiply basis.

Proof. Immediate from Theorem 30 and Proposition 31.

\section{Hadamard method}

In this section we study the Hadamard method, a direct construction of a unitary error basis from a Hadamard matrix. While this is certainly known, we cannot find a clear description of it in full generality, although a special case is worked out in detail in [7]. The main results of this section are Theorem 34, where we show that the quantum shiftand-multiply method contains the Hadamard method as a special case, and Corollary 39, in which we show that this containment is proper. 
Definition 33 (Hadamard method; folklore). For a Hadamard matrix $H$ of order $n$, its associated Hadamard basis $\left\{\left(U_{H}\right)_{i j}\right\}$ is defined as follows:

$$
\left(U_{H}\right)_{i j}=\frac{1}{n} H \circ \operatorname{diag}(H, j)^{\dagger} \circ H^{\dagger} \circ \operatorname{diag}\left(H^{T}, i\right)
$$

Theorem 34. A Hadamard basis is a quantum shift-and-multiply basis.

Proof. By Definition 10 and Theorem 11 we have $\left(U_{H}\right)_{i j}=\left(Q_{H}\right)_{j} \circ \operatorname{diag}\left(H^{T}, i\right)$. Since the transpose of a Hadamard is also a Hadamard, the result follows.

Corollary 35. A Hadamard basis is a unitary error basis.

Proof. Follows from Theorems 20 and 34.

Proposition 36. If two Hadamard matrices are equivalent by Definition 8, then their associated unitary error bases are equivalent by Definition 16.

Proof. We will once again prove equivalence on a case-by-case basis. Again suppose $H^{\prime}=P \circ H$. Then we have the following:

$$
\left(U_{H^{\prime}}\right)_{i j} \stackrel{\text { 3l] }}{=} \frac{1}{n} P \circ H \circ \operatorname{diag}(P \circ H, j)^{\dagger} \circ H^{\dagger} \circ P^{-1} \circ \operatorname{diag}\left(H^{T} \circ P^{-1}, i\right)
$$

Again using the fact that $P$ is real and unitary so, $P^{-1}=P^{\dagger}=P^{T}$. We continue:

$$
\begin{aligned}
& \left(U_{H^{\prime}}\right)_{i j} \stackrel{[15]}{=} \frac{166}{n} P \circ H \circ \operatorname{diag}(H, p(j))^{\dagger} \circ H^{\dagger} \circ P^{-1} \circ \operatorname{diag}\left(a_{p(0), i}, \ldots, a_{p(n-1), i}\right) \\
& \stackrel{27}{=} \frac{1}{n} P \circ H \circ \operatorname{diag}(H, p(j))^{\dagger} \circ H^{\dagger} \circ \operatorname{diag}\left(a_{p^{-1}} p(0), i, \ldots, a_{p^{-1}} p(n-1), i\right) \circ P^{-1} \\
& \underline{\underline{\underline{11}}} \frac{1}{n} P \circ H \circ \operatorname{diag}(H, p(j))^{\dagger} \circ H^{\dagger} \circ \operatorname{diag}\left(H^{T}, i\right) \circ P^{-1} \\
& \stackrel{200}{\sim} \frac{1}{n} H \circ \operatorname{diag}(H, p(j))^{\dagger} \circ H^{\dagger} \circ \operatorname{diag}\left(H^{T}, i\right) \\
& \stackrel{31}{=}\left(U_{H}\right)_{i, p(j)}
\end{aligned}
$$

The case that $H^{\prime}=H \circ P$ is similar. Now suppose $H^{\prime}=D \circ H$, with $D=\operatorname{diag}\left(c_{1}, \ldots, c_{n}\right)$, where $\left|c_{i}\right|=1$. Then we calculate as follows:

$$
\begin{aligned}
\left(U_{H^{\prime}}\right)_{i j} & \stackrel{\text { 311 }}{=} \frac{1}{n} D \circ H \circ \operatorname{diag}(D \circ H, j)^{\dagger} \circ H^{\dagger} \circ D^{\dagger} \circ \operatorname{diag}\left(H^{T} \circ D^{T}, i\right) \\
& \stackrel{17}{=} \frac{1}{n} D \circ H \circ c_{j} \operatorname{diag}(H, j)^{\dagger} \circ H^{\dagger} \circ \operatorname{diag}\left(H^{T} \circ D, i\right) \circ D^{\dagger} \\
& \stackrel{(18)}{=} \frac{c_{j}}{n} D \circ H \circ \operatorname{diag}(H, j)^{\dagger} \circ H^{\dagger} \circ \operatorname{diag}\left(H^{T}, i\right) \circ D \circ D^{\dagger} \\
& \stackrel{\sqrt{201}}{\sim} \frac{1}{n} H \circ \operatorname{diag}(H, j)^{\dagger} \circ H^{\dagger} \circ \operatorname{diag}\left(H^{T}, i\right) \\
& \stackrel{311}{=}\left(U_{H}\right)_{i j}
\end{aligned}
$$

The case $H^{\prime}=H \circ D$ is similar.

Proposition 37 (See [9], Theorem 1). All Hadamard matrices on $\mathbb{C}^{4}$ are equivalent to one of the following Fourier matrices, parameterised by $\alpha \in\left[0, \frac{\pi}{2}\right]$ :

$$
H_{\alpha}:=\left(\begin{array}{cccc}
1 & 1 & 1 & 1 \\
1 & 1 & -1 & -1 \\
1 & -1 & e^{i \alpha} & -e^{i \alpha} \\
1 & -1 & -e^{i \alpha} & e^{i \alpha}
\end{array}\right)
$$

Theorem 38. Every unitary error basis for $\mathbb{C}^{4}$ arising from the Hadamard method is equivalent to a monomial basis. 
Proof. Write $\mathcal{F}_{\alpha}$ for the unitary error basis arising from $H_{\alpha}$ by the Hadamard method, for some fixed $\alpha \in\left[0, \frac{\pi}{2}\right]$. By Propositions 36 and 37 all unitary error bases arising from Hadamards in dimension 4 are equivalent to $\mathcal{F}_{\alpha}$, for some value of $\alpha$. But the following unitary matrix simultaneously monomializes $\mathcal{F}_{\alpha}$, for all values of $\alpha$ :

$$
Y:=\frac{1}{\sqrt{2}}\left(\begin{array}{cccc}
0 & 0 & -1 & 1 \\
-1 & 1 & 0 & 0 \\
0 & 0 & 1 & 1 \\
1 & 1 & 0 & 0
\end{array}\right)
$$

The basis $\mathcal{F}_{\alpha}^{\prime}=\left\{Y \circ F_{i j} \circ Y^{\dagger} \mid F_{i j} \in \mathcal{F}\right\}$ is listed in Section A.2, and is monomial and equivalent to $\mathcal{F}_{\alpha}$. This completes the proof.

Corollary 39. The basis $\mathcal{M}$ of Example 19 is not equivalent to a Hadamard basis.

Proof. Follows from Theorems 30 and 38.

\section{Algebraic method}

Another technique for constructing UEBs is the algebraic method, due to Knill [15]. UEBs obtained using this technique are called nice error bases. The main result in this section is Corollary 44, that the basis $\mathcal{M}$ of Example 19 is not equivalent to a nice error basis. Throughout this section, we use ' $\propto$ ' to denote equality up to multiplication by a unit complex number.

Recall that for a finite group $G$, an $n$-dimensional unitary projective representation is a function $\rho: G \rightarrow U(n)$, valued in the group of $n$-by- $n$ unitary matrices, and for any $g, g^{\prime} \in G$ a complex number $\omega_{g, g^{\prime}} \in \mathbb{C}$ with unit norm, such that we have $\rho\left(g g^{\prime}\right)=\omega_{g, g^{\prime}} \rho(g) \rho\left(g^{\prime}\right)$ and $\rho(1)=\mathbb{I}_{n}$ where 1 is the group identity. We therefore have the following:

$$
\rho(g) \rho\left(g^{\prime}\right) \propto \rho\left(g g^{\prime}\right) \text { for all } g, g^{\prime} \in G
$$

The following result will also be useful.

Lemma 40. Given a unitary projective representation $\rho$ of a group $G$, the following holds:

$$
\rho(g)^{\dagger} \propto \rho\left(g^{-1}\right) \text { for all } g \in G
$$

Proof. As follows: $\rho(g)^{\dagger}=\rho(g)^{\dagger} \rho(1)=\rho(g)^{\dagger} \rho\left(g g^{-1}\right)^{\frac{\sqrt[34]{34}}{\propto}} \rho(g)^{\dagger} \rho(g) \rho\left(g^{-1}\right)=\rho\left(g^{-1}\right)$.

We now give the definition of a nice error basis, and show that a nice error basis is a unitary error basis.

Definition 41 (Nice error basis. See [15], Section 2). Let $G$ be a finite group of order $n^{2}$, and let $\rho$ be an $n$-dimensional unitary projective representation of $G$, such that for all $g \in G$ not equal to the identity, we have the following:

$$
\operatorname{Tr}(\rho(g))=0
$$

Then a nice error basis $\mathcal{R}_{G, \rho}:=\{\rho(g) \mid g \in G\}$ is the image of $\rho$.

Lemma 42 (See [14], Lemma 3). A nice error basis is a unitary error basis. 
We now prove a key proposition, which we will use to establish that our example basis $\mathcal{M}$ of Example 19 is not equivalent to a nice error basis.

Proposition 43. Let $\mathcal{S}$ be a unitary error basis containing the identity matrix $\mathbb{I}_{n}$, such that $\mathcal{S}$ is equivalent to a nice error basis. Then up to multiplication by a unit complex number, $\mathcal{S}$ is closed under taking adjoints.

Proof. Let $\mathcal{R}_{G, \rho}$ be a nice error basis, and let $\mathcal{S}=\left\{c_{g} U \rho(g) V \mid g \in G\right\}$ be an equivalent unitary error basis, with elements $\mathcal{S}_{g}:=c_{g} U \rho(g) V$. Since by hypothesis $\mathbb{I}_{n} \in \mathcal{S}$, there is some $h \in G$ with $\mathcal{S}_{h}=c_{h} U \rho(h) V=\mathbb{I}_{n}$. In particular, writing ' $\propto$ ' to indicate equality up to multiplication by a unit complex number, we have the following:

$$
\begin{aligned}
& \mathbb{I}_{n} \propto U \rho(h) V \\
& \mathcal{S}_{g} \propto U \rho(g) V \text { for all } g \in G
\end{aligned}
$$

We now perform the following calculation, for any $g \in G$ :

$$
\begin{aligned}
& \left(\mathcal{S}_{g}\right)^{\dagger} \stackrel{\sqrt{38}}{\propto} V^{\dagger} \rho(g)^{\dagger} U^{\dagger}=\mathbb{I}_{n} V^{\dagger} \rho(g)^{\dagger} U^{\dagger} \mathbb{I}_{n} \stackrel{\sqrt{37}}{\propto} U \rho(h) V V^{\dagger} \rho(g)^{\dagger} U^{\dagger} U \rho(h) V \\
& =U \rho(h) \rho(g)^{\dagger} \rho(h) V^{\frac{\sqrt{35}}{\propto}} U \rho(h) \rho\left(g^{-1}\right) \rho(h) V^{\stackrel{\sqrt{34}]}{\propto} U \rho\left(h g^{-1} h\right) V^{\frac{\sqrt{387}}{\propto}} \mathcal{S}_{h g^{-1}}}
\end{aligned}
$$

So $\mathcal{S}$ is closed under adjoints, up to multiplication by a unit complex number.

Corollary 44. The basis $\mathcal{M}$ of Example 19 is not equivalent to a nice error basis.

Proof. By inspection of the elements of $\mathcal{M}$, as listed in Section A.1. For a contradiction, let us assume that $\mathcal{M}$ is equivalent to a nice error basis. Note that $\mathcal{M}$ contains the identity matrix; then by Proposition 43, it must be closed under taking adjoints, up to a unit complex number. But this is clearly false: for example, the second element of the first row of $\mathcal{M}_{01}$ has absolute value $\frac{1}{\sqrt{5}}$, but no member of $\mathcal{M}$ has an element with the same absolute value in the second element of the first column.

\section{References}

[1] Samson Abramsky and Bob Coecke. A categorical semantics of quantum protocols. In Proceedings of the 19th Annual IEEE Symposium on Logic in Computer Science, pages 415-425. IEEE, 2004. arXiv:quant-ph/0402130. doi:10.1109/LICS.2004.1319636.

[2] Samson Abramsky and Bob Coecke. Categorical quantum mechanics. Handbook of quantum logic and quantum structures: quantum logic, pages 261-324, 2008.

[3] Somshubhro Bandyopadhyay, Oscar P. Boykin, Vwani Roychowdhury, and Farrokh Vatan. A new proof for the existence of mutually unbiased bases. Algorithmica, 34(4):512-528, 2002. arXiv:quant-ph/0103162. doi:10.1007/s00453-002-0980-7.

[4] Karol Bartkiewicz, Antonín Černoch, Karel Lemr, Adam Miranowicz, and Franco Nori. Experimental temporal steering and security of quantum key distribution with mutually-unbiased bases. arXiv:quant-ph/1503.00612, 2015. 
[5] Ingemar Bengtsson, Wojciech Bruzda, Asa Ericsson, Jan-Ake Larsson, Wojciech Tadej, and Karol Zyczkowski. MUBs and Hadamards of order six. Journal of Mathematical Physics, 48(5):052106, 2007. arXiv:quant-ph/0610161. doi:10.1063/1.2716990.

[6] Charles H Bennett, Gilles Brassard, Claude Crépeau, Richard Jozsa, Asher Peres, and William K Wootters. Teleporting an unknown quantum state via dual classical and Einstein-Podolsky-Rosen channels. Physical Review Letters, 70(13):1895, 1993. doi:10.1103/PhysRevLett.70.1895.

[7] Bob Coecke and Ross Duncan. Interacting quantum observables: categorical algebra and diagrammatics. New Journal of Physics, 13(4):043016, 2011. arXiv:0906.4725. doi:10.1088/1367-2630/13/4/043016.

[8] Bob Coecke and Aleks Kissinger. Quantum computer science, lecture notes, 2013.

[9] Robert Craigen. Equivalence classes of inverse orthogonal and unit Hadamard matrices. Bulletin of the Australian Mathematical Society, 44(01):109-115, 1991. doi:10.1017/s0004972700029506.

[10] Vincenzo D’Ambrosio, Filippo Cardano, Ebrahim Karimi, Eleonora Nagali, Enrico Santamato, Lorenzo Marrucci, and Fabio Sciarrino. Test of mutually unbiased bases for six-dimensional photonic quantum systems. Scientific reports, 3, 2013. arXiv:quant-ph/1304.4081. doi:10.1038/srep02726.

[11] Ronald Aylmer Fisher and Frank Yates. The $6 \times 6$ Latin squares. In Mathematical Proceedings of the Cambridge Philosophical Society, volume 30.04, pages 492-507. Cambridge Univ Press, 1934. doi:10.1017/s0305004100012731.

[12] Sibasish Ghosh and Ajit Iqbal Singh. Invariants for maximally entangled vectors and unitary bases. arXiv:quant-ph/1401.0099, 2014.

[13] Mile Gu, Helen M Chrzanowski, Syed M Assad, Thomas Symul, Kavan Modi, Timothy C Ralph, Vlatko Vedral, and Ping Koy Lam. Observing the operational significance of discord consumption. Nature Physics, 8(9):671-675, 2012. arXiv:quant-ph/1203.0011. doi:10.1038/NPHYS2376.

[14] Andreas Klappenecker and Martin Rötteler. Unitary error bases: Constructions, equivalence, and applications. In Applied Algebra, Algebraic Algorithms and ErrorCorrecting Codes, pages 139-149. Springer, 2003. doi:10.1007/3-540-44828-4_16.

[15] Emanuel Knill. Group representations, error bases and quantum codes. Technical Report LAUR-96-2807, LANL, 1996. arXiv:quant-ph/9608049. doi:10.2172/373768.

[16] Mhlambululi Mafu, Angela Dudley, Sandeep Goyal, Daniel Giovannini, Melanie McLaren, Miles J Padgett, Thomas Konrad, Francesco Petruccione, Norbert Lütkenhaus, and Andrew Forbes. Higher-dimensional orbital-angular-momentumbased quantum key distribution with mutually unbiased bases. Physical Review A, 88(3):032305, 2013. doi:10.1103/physreva.88.032305. 
[17] Benjamin Musto. Exploring quantum teleportation through unitary error bases. Master's thesis, Department of Computer Science, University of Oxford, 2014. arXiv:quant-ph/1608.04608.

[18] Peter Shor. Fault-tolerant quantum computation. In Proceedings of 37th Symposium on Foundations of Computing, pages 56-65. IEEE Computer Society Press, 1996. arXiv:quant-ph/9605011. doi:10.1007/978-0-387-30162-4_143.

[19] Christoph Spengler, Marcus Huber, Stephen Brierley, Theodor Adaktylos, and Beatrix C Hiesmayr. Entanglement detection via mutually unbiased bases. Physical Review A, 86(2):022311, 2012. arxiv:quant-ph/1202.5058. doi:10.1103/physreva.86.022311.

[20] Wojciech Tadej and Karol Życzkowski. A concise guide to complex Hadamard matrices. Open Systems \& Information Dynamics, 13(02):133-177, 2006. arXiv:quant-ph/0512154. doi:10.1007/s11080-006-8220-2.

[21] Reinhard Werner. All teleportation and dense coding schemes. Journal of Physics A: Mathematical and General, 34(35):7081, 2001. arXiv:quant-ph/0003070. doi:10.1088/0305-4470/34/35/332. 


\section{A Lists of unitary error bases}

Here we list the unitary error bases that we make use of in the main text.

\section{A.1 The unitary error basis $\mathcal{M}$}

Here we list the unitary error basis $\mathcal{M}$ defined in Example 19.

$$
\begin{aligned}
& \mathcal{M}_{00}=\left(\begin{array}{llll}
1 & 0 & 0 & 0 \\
0 & 1 & 0 & 0 \\
0 & 0 & 1 & 0 \\
0 & 0 & 0 & 1
\end{array}\right) \quad \mathcal{M}_{01}=\left(\begin{array}{cccc}
0 & \frac{i}{\sqrt{5}} & \frac{2}{\sqrt{5}} & 0 \\
\frac{1}{\sqrt{2}} & 0 & 0 & \frac{1}{\sqrt{2}} \\
-\frac{1}{\sqrt{2}} & 0 & 0 & \frac{1}{\sqrt{2}} \\
0 & \frac{2}{\sqrt{5}} & \frac{i}{\sqrt{5}} & 0
\end{array}\right) \quad \mathcal{M}_{02}=\left(\begin{array}{cccc}
0 & \frac{2}{\sqrt{5}} & \frac{i}{\sqrt{5}} & 0 \\
\frac{1}{\sqrt{2}} & 0 & 0 & \frac{1}{\sqrt{2}} \\
\frac{1}{\sqrt{2}} & 0 & 0 & -\frac{1}{\sqrt{2}} \\
0 & \frac{i}{\sqrt{5}} & \frac{2}{\sqrt{5}} & 0
\end{array}\right) \quad \mathcal{M}_{03}=\left(\begin{array}{llll}
0 & 0 & 0 & 1 \\
0 & 0 & 1 & 0 \\
0 & 1 & 0 & 0 \\
1 & 0 & 0 & 0
\end{array}\right) \\
& \mathcal{M}_{10}=\left(\begin{array}{cccc}
1 & 0 & 0 & 0 \\
0 & i & 0 & 0 \\
0 & 0 & -1 & 0 \\
0 & 0 & 0 & -i
\end{array}\right) \quad \mathcal{M}_{11}=\left(\begin{array}{cccc}
0 & -\frac{1}{\sqrt{5}} & -\frac{2}{\sqrt{5}} & 0 \\
\frac{1}{\sqrt{2}} & 0 & 0 & -\frac{i}{\sqrt{2}} \\
-\frac{1}{\sqrt{2}} & 0 & 0 & -\frac{i}{\sqrt{2}} \\
0 & \frac{2 i}{\sqrt{5}} & -\frac{i}{\sqrt{5}} & 0
\end{array}\right) \quad \mathcal{M}_{12}=\left(\begin{array}{cccc}
0 & \frac{2 i}{\sqrt{5}} & -\frac{i}{\sqrt{5}} & 0 \\
\frac{1}{\sqrt{2}} & 0 & 0 & -\frac{i}{\sqrt{2}} \\
\frac{1}{\sqrt{2}} & 0 & 0 & \frac{i}{\sqrt{2}} \\
0 & -\frac{1}{\sqrt{5}} & -\frac{2}{\sqrt{5}} & 0
\end{array}\right) \quad \mathcal{M}_{13}=\left(\begin{array}{cccc}
0 & 0 & 0 & -i \\
0 & 0 & -1 & 0 \\
0 & i & 0 & 0 \\
1 & 0 & 0 & 0
\end{array}\right) \\
& \mathcal{M}_{20}=\left(\begin{array}{cccc}
1 & 0 & 0 & 0 \\
0 & -1 & 0 & 0 \\
0 & 0 & 1 & 0 \\
0 & 0 & 0 & -1
\end{array}\right) \quad \mathcal{M}_{21}=\left(\begin{array}{cccc}
0 & -\frac{i}{\sqrt{5}} & \frac{2}{\sqrt{5}} & 0 \\
\frac{1}{\sqrt{2}} & 0 & 0 & -\frac{1}{\sqrt{2}} \\
-\frac{1}{\sqrt{2}} & 0 & 0 & -\frac{1}{\sqrt{2}} \\
0 & -\frac{2}{\sqrt{5}} & \frac{i}{\sqrt{5}} & 0
\end{array}\right) \quad \mathcal{M}_{22}=\left(\begin{array}{cccc}
0 & -\frac{2}{\sqrt{5}} & \frac{i}{\sqrt{5}} & 0 \\
\frac{1}{\sqrt{2}} & 0 & 0 & -\frac{1}{\sqrt{2}} \\
\frac{1}{\sqrt{2}} & 0 & 0 & \frac{1}{\sqrt{2}} \\
0 & -\frac{i}{\sqrt{5}} & \frac{2}{\sqrt{5}} & 0
\end{array}\right) \quad \mathcal{M}_{23}=\left(\begin{array}{cccc}
0 & 0 & 0 & -1 \\
0 & 0 & 1 & 0 \\
0 & -1 & 0 & 0 \\
1 & 0 & 0 & 0
\end{array}\right) \\
& \mathcal{M}_{30}=\left(\begin{array}{cccc}
1 & 0 & 0 & 0 \\
0 & -i & 0 & 0 \\
0 & 0 & -1 & 0 \\
0 & 0 & 0 & i
\end{array}\right) \quad \mathcal{M}_{31}=\left(\begin{array}{cccc}
0 & \frac{1}{\sqrt{5}} & -\frac{2}{\sqrt{5}} & 0 \\
\frac{1}{\sqrt{2}} & 0 & 0 & \frac{i}{\sqrt{2}} \\
-\frac{1}{\sqrt{2}} & 0 & 0 & \frac{i}{\sqrt{2}} \\
0 & -\frac{2 i}{\sqrt{5}} & -\frac{i}{\sqrt{5}} & 0
\end{array}\right) \quad \mathcal{M}_{32}=\left(\begin{array}{cccc}
0 & -\frac{2 i}{\sqrt{5}} & -\frac{i}{\sqrt{5}} & 0 \\
\frac{1}{\sqrt{2}} & 0 & 0 & \frac{i}{\sqrt{2}} \\
\frac{1}{\sqrt{2}} & 0 & 0 & -\frac{i}{\sqrt{2}} \\
0 & \frac{1}{\sqrt{5}} & -\frac{2}{\sqrt{5}} & 0
\end{array}\right) \quad \mathcal{M}_{33}=\left(\begin{array}{cccc}
0 & 0 & 0 & i \\
0 & 0 & -1 & 0 \\
0 & -i & 0 & 0 \\
1 & 0 & 0 & 0
\end{array}\right)
\end{aligned}
$$

\section{A.2 The unitary error basis $\mathcal{F}^{\prime}$}

Here we list the unitary error basis $\mathcal{F}^{\prime}$ defined in the proof of Theorem 38 ,

$$
\begin{aligned}
& \mathcal{F}_{00}^{\prime}=\left(\begin{array}{llll}
1 & 0 & 0 & 0 \\
0 & 1 & 0 & 0 \\
0 & 0 & 1 & 0 \\
0 & 0 & 0 & 1
\end{array}\right) \quad \mathcal{F}_{01}^{\prime}=\left(\begin{array}{cccc}
-1 & 0 & 0 & 0 \\
0 & -1 & 0 & 0 \\
0 & 0 & 1 & 0 \\
0 & 0 & 0 & 1
\end{array}\right) \quad \mathcal{F}_{02}^{\prime}=\left(\begin{array}{cccc}
0 & 1 & 0 & 0 \\
e^{-2 i a} & 0 & 0 & 0 \\
0 & 0 & 0 & 1 \\
0 & 0 & 1 & 0
\end{array}\right) \quad \mathcal{F}_{03}^{\prime}=\left(\begin{array}{cccc}
0 & -1 & 0 & 0 \\
-e^{-2 i a} & 0 & 0 & 0 \\
0 & 0 & 0 & 1 \\
0 & 0 & 1 & 0
\end{array}\right)
\end{aligned}
$$

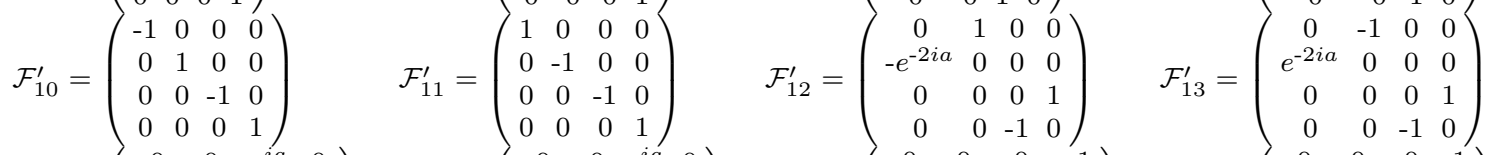

$$
\begin{aligned}
& \mathcal{F}_{20}^{\prime}=\left(\begin{array}{cccc}
0 & 0 & -e^{i a} & 0 \\
0 & 0 & 0 & -1 \\
-e^{i a} & 0 & 0 & 0 \\
0 & -1 & 0 & 0
\end{array}\right) \quad \mathcal{F}_{21}^{\prime}=\left(\begin{array}{cccc}
0 & 0 & e^{i a} & 0 \\
0 & 0 & 0 & 1 \\
-e^{i a} & 0 & 0 & 0 \\
0 & -1 & 0 & 0
\end{array}\right) \quad \mathcal{F}_{22}^{\prime}=\left(\begin{array}{cccc}
0 & 0 & 0 & -1 \\
0 & 0 & -e^{-i a} & 0 \\
0 & -1 & 0 & 0 \\
-e^{i a} & 0 & 0 & 0
\end{array}\right) \quad \mathcal{F}_{23}^{\prime}=\left(\begin{array}{cccc}
0 & 0 & 0 & 0 \\
0 & 0 & e^{-i a} & 0 \\
0 & -1 & 0 & 0 \\
-e^{i a} & 0 & 0 & 0
\end{array}\right) \\
& \mathcal{F}_{30}^{\prime}=\left(\begin{array}{cccc}
0 & 0 & e^{i a} & 0 \\
0 & 0 & 0 & -1 \\
e^{i a} & 0 & 0 & 0 \\
0 & -1 & 0 & 0
\end{array}\right) \quad \mathcal{F}_{31}^{\prime}=\left(\begin{array}{cccc}
0 & 0 & -e^{i a} & 0 \\
0 & 0 & 0 & 1 \\
e^{i a} & 0 & 0 & 0 \\
0 & -1 & 0 & 0
\end{array}\right) \quad \mathcal{F}_{32}^{\prime}=\left(\begin{array}{cccc}
0 & 0 & 0 & -1 \\
0 & 0 & e^{-i a} & 0 \\
0 & -1 & 0 & 0 \\
e^{i a} & 0 & 0 & 0
\end{array}\right) \quad \mathcal{F}_{33}^{\prime}=\left(\begin{array}{cccc}
0 & 0 & 0 & 1 \\
0 & 0 & -e^{-i a} & 0 \\
0 & -1 & 0 & 0 \\
e^{i a} & 0 & 0 & 0
\end{array}\right)
\end{aligned}
$$

